\title{
Solvency II: Setting the Pace for Regulatory Change
}

\author{
Matthew Elderfield \\ Bermuda Monetary Authority, BMA House, 43 Victoria Street, Hamilton HM12, Bermuda. \\ E-mail: melderfield@bma.com
}

The international regulatory environment for the insurance sector is experiencing an intense period of change. The issue of maintaining compliance with international standards has become an increasing priority for insurance regulators worldwide. In recent years the movement toward the global standardisation of insurance regulation has gained momentum, as the industry itself has become more globalised. The significant market turmoil over the past year that has had worldwide impact on the financial sector has added another complex dimension to this environment. This paper argues that there are three key regulatory trends that will impact the insurance industry in the short- to medium-term, based on two underlying key drivers of regulatory change over this time horizon. These drivers can be put simply and should be no surprise. They are: first, Solvency II and second, sub prime.

The Geneva Papers (2009) 34, 35-41. doi:10.1057/gpp.2008.35

Keywords: key insurance regulatory trends; Solvency II

\section{Introduction}

Through its development of Solvency II, the European Union (EU) has emerged as the principal catalyst of regulatory change in the insurance industry. I think this is for two reasons:

First, simply put, the EU has a decision-making process that allows it to proceed without requiring full consensus. These procedures may be highly complicated and ornate at times, but they operate by qualified majority voting and provide a mechanism for difficult policy areas to be transposed into actual legal requirements, which are binding on member states and individual firms. In contrast, the other insurance superpower, the United States, cannot easily achieve a similar level of dynamism for decision-making.

Second, the EU is now setting the pace because of the ambition of the agenda in Solvency II. The directive is no mere codification of existing practice or tidying up of rules. It is not a Xerox of the Basel banking legislation across the insurance industry. Indeed, in some areas - group supervision and the use of internal models in particular - it represents a step change in financial services regulation.

If Solvency II is the main source of change, one should not ignore the effects of sub prime on the insurance industry.

The most authoritative regulatory action plan on sub prime has come from the Financial Stability Forum (FSF). While there is scant mention of insurance in this 
document, ${ }^{1}$ the programme initiated by the FSF will have important implications for insurers:

- First, and most obviously, the FSF calls for tighter solvency standards for financial guaranty companies.

- Second, the FSF also calls for accelerated work by the International Accounting Standards Board on accounting for financial instruments; this will accelerate and crystallise the debate on fair value.

- Third, there is a strong prospect that whatever new rules emerge for bankers, say for more robust risk management, they will eventually feed their way into standards for insurers. The transmission mechanisms will be concerns over regulatory arbitrage, level playing fields and capital market convergence with insurance.

- Fourth, the work of the FSF will reinforce key elements of the agenda in Solvency II. FSF has called for supervisory colleges - that is meetings between all the regulators involved in a single group. Solvency II requires supervisory colleges under its group supervision rules. The FSF encourages greater disclosure and investor scrutiny; Solvency II does similarly through its Pillar 3 framework. In other words, in certain key areas of supervision and risk management standards, the insurance industry faces two major forces pushing simultaneously in the same direction.

- Finally, and more long-term, sub prime has acted as the trigger for a serious debate about regulatory structure in the United States. Predicting the outcome of this debate is only marginally less perilous than predicting when sub prime write-downs will cease. Treasury Secretary Paulson's plan is clearly only the opening shot in this debate. But it also looks like a debate that could result in real change at the end of the day. If it touches on insurance regulation, as seems likely, the decision-making dynamic spoken about earlier, might shift.

\section{Group supervision and risk management: new regulatory challenges}

With Solvency II and sub prime as the main drivers of regulatory change, the three key trends that will have short-to-medium impact on the insurance industry are:

- the development of enhanced supervisory requirements for insurance groups;

- increased scrutiny of risk management practice, while allowing greater scope for the use of internal economic capital models;

- increased disclosure of risk metrics.

The regulatory changes being proposed in these areas are, in my opinion, innovative and challenging for both the industry and regulators alike.

Starting with group supervision, this can be defined broadly as two things: requiring insurance groups to calculate their solvency requirements on a consolidated group basis, as an additional solvency test to that which must be met at an individual legal

\footnotetext{
${ }^{1}$ Report of the Financial Stability Forum on Enhancing Market and Institutional Resilience (7 April 2008).
} 
entity level. Group supervision also refers to cooperation between regulators, in some form or another, to ensure that all the risks in a group are adequately monitored in a coordinated manner.

Even before the regulator enters the equation, it is clear that group issues are important. Insurance firms are often operating on a global scale, across a range of markets via subsidiaries, branches and cross-border transactions. Such groups are increasingly subject to integrated risk and capital management, seeking to optimise efficient capital allocation and ensure universal applicability of key controls. Acquisitions and consolidation, which may accelerate due to a soft market, means legacy structures are bolted together and tough decisions are needed about new structures. As a result, group structure and controls are an important element of strategy, to be adjusted to optimise performance.

Over the next few years, there will be a new element: the supervisory arrangements for groups will change in important ways to add a new variable into this difficult mix of considerations.

At present, many supervisors only regulate at the legal entities within their jurisdiction. Indeed, while the International Association of Insurance Supervisors has a standard calling for supervision at a group level, this is the least observed of all such IAIS standards.

All that is going to change, because Solvency II does three things:

First, it strengthens group supervision in general terms. While there have been elements of group supervision in existing EU directives, the new directive sets out a more elaborate and exacting framework of how solvency requirements are calculated, and how supervisors must work with one another.

Second, and of fundamental importance, Solvency II is looking to set out a mechanism whereby capital requirements for local legal entities need not be locked into those jurisdictions. Instead, surplus capital may be held at the centre, at the group level and count towards local requirements. This is a very significant innovation beyond the capital adequacy framework for banks. This provides scope for more efficient capital management by groups. These requirements are controversial and not yet agreed, but if they do emerge in the final directive in one form or another, they will be a very important innovation in financial regulation.

Third, and of crucial importance to anyone outside of the EU, Solvency II effectively exports the broad outlines of this regime. In order to gain access to EU markets on non-discriminatory terms, firms from non-EU jurisdictions such as Bermuda and the United States will be subject to a mutual recognition assessment to see if our rules are broadly equivalent. While there is flexibility to some degree, there needs to be something in the group supervision space, where for many jurisdictions there has been nothing before.

In Bermuda, we have already recognised the importance of developing a framework for group supervision. It makes sense as a regulator, to allow the Bermuda Monetary Authority (BMA) to get a more complete picture of the risks in the firms we regulate, as well as making sense to the industry, in terms of EU market access and having more coordinated regulatory interaction. The BMA will publish a discussion paper on group supervision in the first quarter of 2009 with a view to implementing group supervision rules by 2011. In the meantime, we have geared up our level of interaction with 
overseas regulators and hosted a series of pilot supervisory colleges for our largest insurance groups.

In summary, the main consequences of the changes being proposed with respect to group supervision are that:

- the regulatory framework is changing and will impose new requirements to calculate solvency on a consolidated group basis;

- the particular group structure of insurance companies and the extent to which they have an integrated risk management and capital allocation framework could shortly become an important strategic advantage because of these new rules;

- by working with their lead supervisor, firms have the prospect of a more coordinated dialogue with the various regulators around their group.

The second major trend relates to risk management practice and economic capital modelling.

The simple and perhaps obvious point here is that the combination of Solvency II and sub prime will lead to increased regulatory scrutiny of the risk management framework of insurance companies. The qualitative supervisory review of risk management arrangements is hard wired into Solvency II through the so-called second Pillar and the importance of this review has been reinforced by the lessons of sub prime, as the degree of supervisory challenge of firms risk management has had to step up a gear. There is also a conclusion that the quantitative calculation of the Pillar 1 framework - that is the actual solvency formulae - needs to be supplemented by the insurer itself conducting a holistic assessment of risk. In Solvency II this is called the "Own Risk and Solvency Assessment". One might like to think of this as a regulator-mandated Enterprise Risk Management exercise.

This assessment will be subject to qualitative supervisory review. And this qualitative review has a hard edge to it, as supervisors can use the Pillar 2 framework to assess supplemental individual capital requirements where they are not satisfied with an insurer's standards.

In Bermuda, our approach to this area is to require qualitative risk management information to be submitted as a supplement to our new Bermuda Solvency Capital Requirement (BSCR). The BSCR is equivalent to the Solvency II standardised capital framework and the supplementary risk management information provides data to support our qualitative supervisory review. Under our framework, this review permits the BMA to specify capital add-ons if we feel that risks have not been accounted for appropriately. Our qualitative assessment is also based on stress testing information. The BMA requires firms to provide information on two specified macroeconomic stress scenarios plus their three most adverse realistic disaster scenarios. We will conduct peer review analysis of this information to identify outliers for further supervisory dialogue.

While these are important changes at the EU and Bermuda level, an even more important regulatory innovation will come with the recognition of firm's own internal economic capital models as an alternative to the use of standardised solvency formulae. This creates an incentive to use internal models to optimise regulatory capital levels, much like exists in the Basel framework for banks. But here as well we see a fundamental innovation in Solvency II compared to Basel. In the case of 
Basel, the regulatory framework prescribed limits on the extent to which diversification effects can be recognised by prescribing correlations to be used with models. Solvency II does not place such constraints on modelling but will likely allow firms to propose their own models, with full recognition of diversification, provided they can be justified to their supervisors. Combined with the group supervision framework, these diversification effects can have powerful benefits for group capital requirements.

While there are regulatory benefits of encouraging diversification, a source of concern is that insurers may not take sufficient account of the impact of stressed market conditions or unexpected events on correlations and that regulators may have insufficient intellectual capital to argue the fine points of these correlations with their firms. It is important to be cautious about recognising unlimited diversification effects unless firms have conducted rigorous analysis of the impacts of stress events on correlations.

At the BMA, we are surveying economic capital modelling practice in the Bermuda market and conducting a series of on-site visits to familiarise ourselves with the state of play among a sample of firms. Based on this work we are planning to issue entry criteria for the use of modelling as an alternative to our solvency rules.

The take-away from all this is quite straightforward: that the quality of a firm's risk management framework will be an even more important issue for regulators; that insurers will need to embrace an Enterprise Risk Management framework; that this overall framework will be assessed by their regulator and deficiencies may lead to more onerous solvency charges compared to those applied to competitors; and finally that embracing economic capital modelling may, within reason, provide a strategic advantage to optimise a firm's regulatory capital.

\section{Transparency and disclosure: universal tools for market discipline}

Both Solvency II and sub prime are pushing firmly in the same direction: enhanced disclosure of financial information and risk metrics in order to provide investors with additional information to enhance market discipline. In Solvency II, this comes through the Pillar 3 framework, which requires firms to publish specified financial data, solvency calculations, and qualitative and quantitative risk management information. In the work of the FSF, there is a general exhortation to improve disclosure, accelerate work on fair value and to have less reliance on rating agencies.

The issue of the influence of rating agencies is interesting and challenging. Companies of course recognise the importance of meeting regulatory requirements and required regulatory capital while conducting their business. However, it is normally rating agency capital, which they regard as the constraint on their business and which is of principal concern. The trends I have described here will present important strategic opportunities and real costs on firms regardless of the presence of the rating agencies. However, one cannot ignore the influence of rating agencies overall on the markets and on the standing of individual companies within those markets. 
Criticising the rating agencies has been easy sport in recent times. But the hard truth is that if the rating agencies were found to have had too much influence in the structured credit market - and were relied upon by investors too blindly - all with unfortunate repercussions, then surely the same problems exist in the insurance market. All too often, firms engage in strategic contortionism and financial gymnastics at the behest of the rating agencies.

The regulatory agenda described above does in fact offer a partial prospect of rebalancing the influence of the agencies in the insurance industry. Increased scrutiny on risk management provides an opportunity for regulators to assess whether firms are blindly following credit ratings or are conducting their own due diligence.

But more importantly, the combination of internationally harmonised solvency standards, recognition of economic capital modelling and enhanced disclosure holds out the prospect of a universal set of insurance solvency and risk metrics, which can supplement the rating agency rating. The banking world has the Basel capital ratio as a common and public metric that is the focus of analysts (and indeed rating agencies). And by getting a regulatory seal of approval of sorts for their economic capital models, firms have a stronger basis for dialogue with the rating agencies on economic capital. The regulatory capital framework provides a common language for dialogue with investors and rating agencies, which means the rating agencies own proprietary model is no longer the only game in town.

\section{Preparation: the priority for continued, dynamic regulatory change}

The trends described here will pose significant challenges to the industry and regulators alike. At the BMA, we are in a period of significant growth in our resources in order to meet these challenges. The BMA continues to add to staffing levels yearon-year and has recently formed an in-house actuarial team, a specialist unit to deal with non-compliant firms and an independent risk and policy unit. We also engage in outsourcing arrangements with professional services firms where we feel we need to supplement our resources.

We are also investing considerably in learning and development and have increased our training budget significantly this year. In addition to sponsoring study with professional bodies, we are developing an extensive in-house curriculum and this year have, for example, facilitated supervisory case study training for our on-site staff and various training modules on economic capital modelling.

In addition, we are well advanced in our work on mutual recognition. Our starting point is one of high compliance with international standards. But by rolling out our programme of enhancements to the solvency regime for commercial insurers - with initiatives such as the BSCR, exploring the use of economic capital modelling and looking at solvency on a group basis - we are not resting on our laurels.

\section{Reference}




\section{About the Author}

Matthew Elderfield is the Chief Executive Officer of the Bermuda Monetary Authority (BMA). He has over 20 years of experience in international financial services regulation, encompassing supervision, policy and risk management and high-level advocacy in the United States and Europe. Prior to working at the BMA, he spent 8 years at the Financial Services Authority, where he held a series of senior management posts. He has a master's degree in International Relations from Cambridge University. 